\title{
ENERGY RELATIONS FOR SH WAVES INTERACTING WITH A FRICTIONAL CONTACT INTERFACE
}

\author{
Eugene L. Chez and John Dundurs \\ Department of Civil Engineering, Northwestern University, Evanston, IL 60201, U.S.A. \\ and \\ Maria Comninou \\ Department of Civil Engineering, University of Michigan, Ann Arbor, MI 48109, U.S.A.
}

(Received 23 March 1982; in revised form 5 November 1982)

\begin{abstract}
Ahetract-The paper considers the partition of energy when an incident SH wave is reflected and refracted by a fricitonal contact interface that slips. The effect of applied shear tractions which cause a slow creeping motion is included in the analysis.
\end{abstract}

\section{INTRODUCTION}

The reflection and refraction of horizontally polarized shear (SH) waves by a frictional contact interface that can slip has been studied by the present authors [1-3] and Miller [4-6]. If the solids are sheared, the localized slip induced by the waves is more pronounced in one direction than in the other, which leads to the interesting phenomenon of the solids creeping with respect to each other in spite of the fact that no global slip takes place [1]. The applied shear tractions do work on the creeping motion, and it is pertinent to ask whether all of this work is dissipated by friction or, conceivably, some of it is transferred to the wave motion so that the reflected and refracted waves carry more energy than the incident wave.

\section{BASIC RELATIONS}

The physical situation is depicted in Fig. 1. Two solids are pressed together and at the same time sheared without causing catastrophic slip. The incident SH wave $(n=0)$ is reflected $(n=1)$ and refracted $(n=2)$. The solution for the wave motion under localized slip is constructed by correcting the fields for the welded interface. The latter corresponds to the displacements[7]

$$
u_{3}^{(n)}=\operatorname{Re}\left\{C_{n} d_{3}{ }^{(n)} \exp \left(i y_{n}\right)\right\}, \quad n=0,1,2
$$

where

$$
\begin{aligned}
& y_{n}=k_{n}\left[\mathbf{x} \cdot \mathbf{p}^{(n)}-c_{n} t\right] \\
& C_{n}=A_{n}+i B_{n}
\end{aligned}
$$

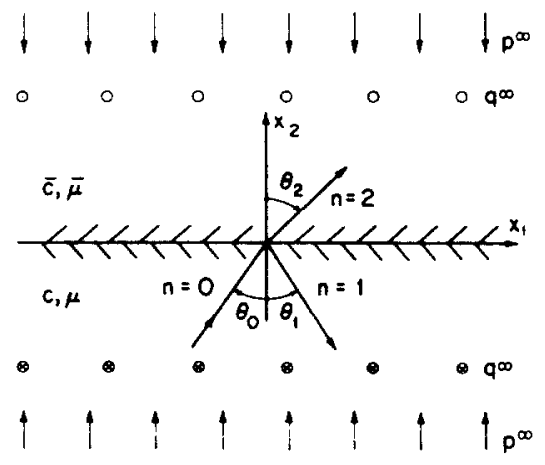

Fig. 1. Incident $(n=0)$, reflected $(n=1)$ and refracted $(n=2)$ waves. 
$C_{0}$ is real, and $\mathbf{d}^{(n)}$ and $\mathbf{p}^{(n)}$ are unit vectors defining the direction of motion and direction of propagation of the incident, reflected and refracted SH waves. The displacements (1) yield the interfacial shear tractions

$$
\left[\sigma_{23}^{(0)}+\sigma_{23}^{(1)}\right]_{x_{2}=0}=\left[\sigma_{23}^{(2)}\right]_{x_{2}=0}=\operatorname{Re}\left\{i A_{0} \exp (i \eta)\right\}
$$

where

$$
\begin{gathered}
\mathscr{A}_{0}=\mu C_{0} k_{0} \frac{2 \Gamma \cos \theta_{0} \cos \theta_{2}}{\gamma \cos \theta_{0}+\Gamma \cos \theta_{2}} \\
\Gamma=\bar{\mu} / \mu, \quad \gamma=\bar{c} / c
\end{gathered}
$$

and

$$
\eta=k_{0}\left(x_{1} \sin \theta_{0}-c t\right)
$$

can be viewed as a coordinate moving along the inteface with the velocity $c / \sin \theta_{0}$.

The corrective solution is taken as the additional displacement associated with the reflected and refracted waves [1]

$$
v_{3}^{(n)}=\operatorname{Re}\left\{\sum_{m=1}^{\infty} F_{m}^{(n)} \exp \left(i m y_{n}\right)\right\}, \quad n=1,2
$$

where

$$
F_{m}^{(n)}=D_{m}^{(n)}+i E_{m}^{(n)}
$$

In order to account for the global creeping motion between the two solids, we also add to the displacements of the upper and lower solids the terms $\frac{1}{2} U t$ and $-\frac{1}{2} U t$, respectively, where $U$ is the velocity of the creeping motion. The requirement that the shear tractions be continuous at the interface yields

$$
F_{m}^{(2)}=-F_{m}^{(1)} \frac{\gamma \cos \theta_{0}}{\Gamma \cos \theta_{2}}
$$

For subcritical angles of incidence, or $\sin \theta_{0}<c / \bar{c}$ which avoids total reflection, the slip velocity and shear tractions at the interface are

$$
\begin{aligned}
V(\eta)=\left[\dot{v}_{3}^{(2)}-\dot{v}_{3}^{(1)}\right]_{x_{2}=0}=U-k_{0} c b \sum_{m=1}^{\infty} m\left[D_{m}{ }^{(1)} \sin m \eta+E_{m}{ }^{(1)} \cos m \eta\right] \\
S(\eta)=q^{\infty}+\left[\sigma_{23}^{(0)}+\sigma_{23}^{(1)}+\tau_{23}^{(1)}\right]_{x_{2}=0}=q^{\infty}+\left[\sigma_{23}^{(2)}+\tau_{23}^{(2)}\right]_{x_{2}=0} \\
=q \infty-\mathscr{A}_{0} \sin \eta+\mu a \sum_{m=1}^{\infty} m\left[D_{m}^{(1)} \sin m \eta+E_{m}{ }^{(1)} \cos m \eta\right]
\end{aligned}
$$

where $\tau_{23}^{(n)}$ are the stresses derived from (6), and

$$
a=k_{0} \cos \theta_{0}, \quad b=1+\frac{\gamma \cos \theta_{0}}{\Gamma \cos \theta_{2}}
$$

It may be noted that from (9)

$$
U=\frac{1}{2 \pi} \int_{-\pi}^{\pi} V(\eta) \mathrm{d} \eta
$$


The distribution of the shear tractions and the slip velocity are shown in Fig. 2. Due to the periodicity of the incident harmonic wave it is sufficient to consider only the representative interval $-\pi<\eta<\pi$. In the left slip zone $V(\eta)>0$, and

$$
S(\eta)=f p^{\infty}, \quad \alpha_{1}<\eta<\beta_{1}
$$

where $f$ denotes the friction coefficient. In the right slip zone $V(\eta)<0$, and

$$
S(\eta)=-f p^{\infty}, \quad \alpha_{2}<\eta<\beta_{2} .
$$

The rest of the representative interval consists of stick zones in which

$$
V(\eta)=0, \quad-\pi<\eta<\alpha_{1} \quad \beta_{1}<\eta<\alpha_{2} \text { and } \beta_{2}<\eta<\pi .
$$

The boundary conditions (13)-(15) combined with (9) and (10) lead to the result that

$$
\sum_{m=1}^{\infty} m\left[D_{m}^{(1)} \sin m \eta+E_{m}^{(1)} \cos m \eta\right]=G(\eta)
$$

where

$$
\begin{aligned}
G(\eta) & =\frac{1}{\mu a}\left(\mathscr{A}_{0} \sin \eta+f p^{\infty}-q^{\infty}\right), \quad \alpha_{1}<\eta<\beta_{1} \\
& =\frac{1}{\mu a}\left(\mathscr{A}_{0} \sin \eta-f p^{\infty}-q^{\infty}\right), \quad \alpha_{2}<\eta<\beta_{2} \\
& =\frac{U}{k_{0} c b}, \quad-\pi<\eta<\alpha_{1}, \beta_{1}<\eta<\alpha_{2} \text { and } \beta_{2}<\eta<\pi
\end{aligned}
$$

\section{Consequently}

$$
S(\eta)=q^{\infty}+\frac{h \mu U}{c}-\alpha_{0} \sin \eta, \quad-\pi<\eta<\alpha_{1}, \quad \beta_{1}<\eta<\alpha_{2} \text { and } \beta_{2}<\eta<\pi
$$

in the stick zones, and

$$
\begin{aligned}
V(\eta) & =U-\frac{c}{h \mu}\left(\mathscr{A}_{0} \sin \eta+f p^{\infty}-q^{\infty}\right), \quad \alpha_{1}<\eta<\beta_{1} \\
& =U-\frac{c}{h \mu}\left(\mathscr{A}_{0} \sin \eta-f p^{\infty}-q^{\infty}\right), \quad \alpha_{2}<\eta<\beta_{2}
\end{aligned}
$$

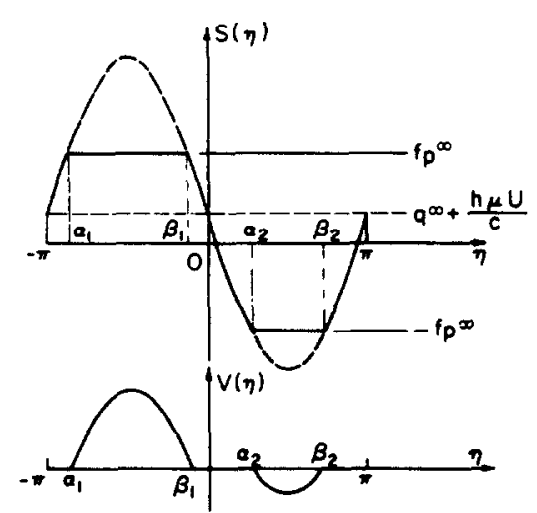

Fig. 2. Interface shear tractions and slip velocity. 
in the slip zones, where

$$
h=\frac{a}{k_{0} b}=\frac{\Gamma \cos \theta_{0} \cos \theta_{2}}{\gamma \cos \theta_{0}+\Gamma \cos \theta_{2}}
$$

Since (16) is a Fourier series,

$$
\begin{aligned}
& D_{m}^{(1)}=\frac{1}{\pi m} \int_{-\pi}^{\pi} G(\eta) \sin m \eta \mathrm{d} \eta \\
& E_{m}^{(1)}=\frac{1}{\pi m} \int_{-\pi}^{\pi} G(\eta) \cos m \eta \mathrm{d} \eta
\end{aligned}
$$

The intervals occupied by the slip zones are determined on basis of the inequalities

$$
\begin{aligned}
V(\eta)>0, & \alpha_{1}<\eta<\beta_{1} \\
& <0, \quad \alpha_{2}<\eta<\beta_{2} .
\end{aligned}
$$

The slip zones are centered on $\eta= \pm \pi / 2$, so that

$$
\alpha_{1}=-\left(\pi+\beta_{1}\right), \quad \beta_{2}=\pi-\alpha_{2}
$$

and in the general case of two slip zones

$$
\begin{gathered}
\sin \beta_{1}=-\frac{1}{\mathscr{A}_{0}}\left(f p^{\infty}-q^{\infty}-\frac{h \mu U}{c}\right),-\pi / 2<\beta_{1}<\pi / 2 \\
\sin \alpha_{2}=\frac{1}{\mathscr{A}_{0}}\left(f p^{\infty}+q^{\infty}+\frac{h \mu U}{c}\right), \quad 0<\alpha_{2}<\pi / 2 .
\end{gathered}
$$

Moreover, (16) yields

$$
\frac{h \mu U}{c}\left(\alpha_{2}-\beta_{1}\right)=\mathscr{A}_{0}\left(\cos \beta_{1}-\cos \alpha_{2}\right)-f p^{\infty}\left(\beta_{1}+\alpha_{2}\right)+q^{\infty}\left(\pi+\beta_{1}-\alpha_{2}\right)
$$

The system (25)-(27) must be solved for $\beta_{1}, \alpha_{2}$ and $U$ by iteration. The right slip zone disappears when the last three equations predict $\sin \alpha_{2}$ to be larger than 1. In such case (27) must be modified by setting $\alpha_{2}=\pi / 2$.

\section{ENERGY PARTITION AND DISSIPATION}

The power or rate of work per unit area is the inner product of tractions and the particle velocity [7]. Consider a thin slice of material containing the interface. Using (5) it follows that the power averaged either over a wave length or a period of the incident wave is

$$
P_{-}=\left.\frac{1}{2 \pi} \int_{-\pi}^{\pi}\left[q^{\infty}+\sigma_{23}^{(0)}+\sigma_{23}^{(1)}+\tau_{23}^{(1)}\right]\left[-\frac{1}{2} U+\dot{u}_{3}^{(0)}+\dot{u}_{3}^{(1)}+\dot{v}_{3}^{(1)}\right]\right|_{x_{2}=0-} \mathrm{d} \eta
$$

on the bottom surface and

$$
P_{+}=\left.\frac{1}{2 \pi} \int_{-\pi}^{\pi}\left[q^{\infty}+\sigma_{23}^{(2)}+\tau_{23}^{(2)}\right]\left[\frac{1}{2} U+\dot{u}_{3}^{(2)}+\dot{v}_{3}^{(2)}\right]\right|_{x_{2}=0+} \mathrm{d} \eta
$$

on the top surface of the slice. The power dissipated by friction at the interface is

$$
P_{d}=\frac{1}{2 \pi} \int_{-\pi}^{\pi} S(\eta) V(\eta) \mathrm{d} \eta
$$


Clearly we must have

$$
P_{-}+P_{+}=P_{d}
$$

which can be used as a check on the calculations.

Substituting the stresses and velocities derived from (1) and (6) into (28), it is seen that what might be called cross terms do not contribute to the integral, and

$$
P_{-}=\left.\frac{1}{\pi} \int_{-\pi}^{\pi}\left\{\frac{1}{2} q^{\infty} U-\sigma_{23}^{(0)} \dot{u}_{3}^{(0)}-\left[\sigma_{23}^{(1)}+\tau_{23}^{(1)}\right]\left[\dot{u}_{3}^{(1)}+\dot{v}_{3}^{(1)}\right]\right\}\right|_{x_{2}=0-} \mathrm{d} \eta
$$

Similarly from (29)

$$
P_{+}=\left.\frac{1}{2 \pi} \int_{-\pi}^{\pi}\left\{\frac{1}{2} q^{\infty} U+\left[\sigma_{23}^{(2)}+\tau_{23}^{(2)}\right]\left[\dot{u}_{3}^{(2)}+\dot{v}_{3}^{(2)}\right]\right\}\right|_{x_{2}=0+} d \eta
$$

The various terms in (32) and (33) admit a direct interpretation. The power input of the applied shear tractions is

$$
P_{q}=q^{\infty} U
$$

whereas the input of the incident wave is

$$
P_{0}=-\frac{1}{2 \pi} \int_{-\pi}^{\pi} \sigma_{23}^{(0)} \dot{u}_{3}^{(0)} \mathrm{d} \eta
$$

The power extracted from the slice by the reflected wave is

$$
P_{1}=\frac{1}{2 \pi} \int_{-\pi}^{\pi}\left[\sigma_{23}^{(1)}+\tau_{23}^{(1)}\right]\left[\dot{u}_{3}{ }^{(1)}+\dot{v}_{3}{ }^{(1)}\right] \mathrm{d} \eta
$$

and that of the refracted wave

$$
P_{2}=-\frac{1}{2 \pi} \int_{-\pi}^{\pi}\left[\sigma_{23}^{(2)}+\tau_{23}^{(2)}\right]\left[\dot{u}_{3}^{(2)}+\dot{v}_{3}^{(2)}\right] d \eta .
$$

In terms of these symbols, (31) becomes

$$
P_{0}+P_{q}=P_{1}+P_{2}+P_{d} .
$$

It may be noted that $P_{1}, P_{2}$ and $P_{d}$ are taken as positive for negative work done on the material contained in the slice.

The remaining task is to carry out the integrations using (16) and (17). The calculations are tedious, but straightforward, and it suffices to simply give the results. Thus, also taking advantage of (28),

$$
\begin{gathered}
P_{0}=\frac{1}{2} C_{0}^{2} \mu k_{0} c a \\
\frac{P_{a}}{P_{0}}=\frac{8 q^{\infty}}{A_{0} b\left(\alpha_{2}-\beta_{1}\right)}\left\{\cos \beta_{1}-\cos \alpha_{2}-\frac{f p^{\infty}}{A_{0}}\left(\alpha_{2}+\beta_{1}\right)+\frac{q^{\infty}}{d_{0}}\left(\pi-\alpha_{2}+\beta_{1}\right)\right\} \\
\frac{P_{1}}{P_{0}}=\left(\frac{b-2}{b}\right)^{2}+\frac{4(b-1)}{\pi b^{2}}\left\{\pi-\alpha_{2}+\beta_{1}+\frac{1}{2}\left(\sin 2 \alpha_{2}-\sin 2 \beta_{1}\right)+\frac{2\left(\cos \beta_{1}-\cos \alpha_{2}\right)^{2}}{\alpha_{2}-\beta_{1}}\right\} \\
-\frac{8}{b\left(\alpha_{2}-\beta_{1}\right)}\left\{\frac{2 f p^{\infty}}{\pi A_{0}}\left(\alpha_{2} \cos \beta_{1}-\beta_{1} \cos \alpha_{2}\right)-\frac{q^{\infty}}{A_{0}}\left(\cos \beta_{1}-\cos \alpha_{2}\right)\right\}
\end{gathered}
$$




$$
+\frac{8}{b^{2}\left(\alpha_{2}-\beta_{1}\right)}\left\{\frac{1}{\pi}\left(\frac{f p^{\infty}}{\mathscr{A}_{0}}\right)^{2}\left[\pi\left(\alpha_{2}-\beta_{1}\right)+4 \alpha_{2} \beta_{1}\right]-\frac{2 f p^{\infty} q^{\infty}}{\mathscr{A}_{0}^{2}}\left(\alpha_{2}+\beta_{1}\right)+\left(\frac{q^{\infty}}{\mathscr{A}_{0}}\right)^{2}\left(\pi-\alpha_{2}+\beta_{1}\right)\right\}
$$

$$
\begin{aligned}
\frac{P_{2}}{P_{0}}= & \frac{4(b-1)}{\pi b^{2}}\left\{\alpha_{2}-\beta_{1}-\frac{1}{2}\left(\sin 2 \alpha_{2}-\sin 2 \beta_{1}\right)-\frac{2\left(\cos \beta_{1}-\cos \alpha_{2}\right)^{2}}{\alpha_{2}-\beta_{1}}\right\} \\
& +\frac{8(b-1)}{b^{2}\left(\alpha_{2}-\beta_{2}\right)}\left\{\frac{1}{\pi}\left(\frac{f p^{\infty}}{\mathscr{A}_{0}}\right)^{2}\left[\pi\left(\alpha_{2}-\beta_{1}\right)+4 \alpha_{2} \beta_{1}\right]-\frac{2 f p^{\infty} q^{\infty}}{\mathscr{A}_{0}^{2}}\left(\alpha_{2}+\beta_{1}\right)+\left(\frac{q^{\infty}}{\mathscr{A}_{0}}\right)^{2}\left(\pi-\alpha_{2}+\beta_{1}\right)\right\} \\
& \frac{P_{d}}{P_{0}}=\frac{8 f p^{\infty}}{\pi \mathscr{A}_{0} b\left(\alpha_{2}-\beta_{1}\right)}\left\{2\left(\alpha_{2} \cos \beta_{1}-\beta_{1} \cos \alpha_{2}\right)-\frac{f p^{\infty}}{\mathscr{A}_{0}}\left[\pi\left(\alpha_{2}-\beta_{1}\right)+4 \alpha_{2} \beta_{1}\right]+\frac{\pi q^{\infty}}{\mathscr{A}_{0}}\left(\alpha_{2}+\beta_{1}\right)\right\} .
\end{aligned}
$$

In case there is only one slip zone, (40)-(43) must be modified by setting $\alpha_{2}=\pi / 2$.

The expressions obtained which give the energy partition between the reflected and refracted waves and the rate of energy dissipation are too complicated for reaching general conclusions, and the effect of the applied tractions resulting in the creeping motion must be studied on basis of specific examples.

\section{EXAMPLES}

It is seen from (25) to (27) and (40) to (43) that the power ratios depend on $f p^{\infty} / \mathscr{A}_{0}, q^{\infty} / \mathscr{A}_{0}$ and $b$ which is given by (11). Thus the material constants and the angle of incidence enter the results only through the single parameter $b$.

It is convenient to plot the power ratios versus $f p^{\propto} / \mathscr{A}_{0}$ for selected values of $q^{\infty} / \mathscr{A}_{0}$ and $b$. Only the range $q^{\infty}<f p^{\infty}<q^{\infty}+\mathscr{A}_{0}$ is covered in the diagrams, since $f p^{\infty}<q^{\infty}$ corresponds to catastrophic slip, and $q^{\infty}+\mathscr{A}_{0}<f p^{\infty}$ to no slip at all with the interface acting as if it were welded. The values selected for plotting are $q^{\infty} / \mathscr{A}_{0}=0,0.1,0.4$ and 0.8 with $b=1,2$ and 4 . The value of $b=1$ corresponds to the lower solid being in contact with a rigid upper solid $(\Gamma=\infty)$. There is no refracted wave for this case. The value of $b=2$ corresponds to identical materials, whereas $b=4$ can be achieved when the upper solid has a much lower shear modulus than the lower solid. The diagrams for the power ratios are shown in Figs. 3-6 (the curves in the figures are labeled with the subscripts of the power ratios, e.g. $d$ denotes the curves of $P_{d}\left(P_{0}\right)$.
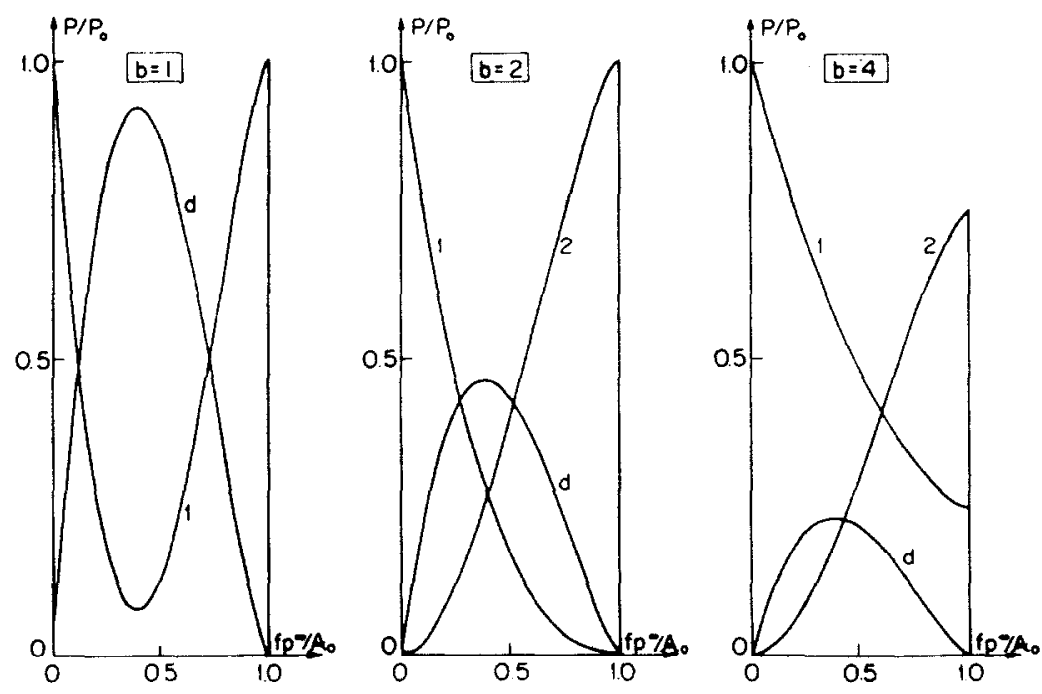

Fig. 3. Power ratios for $q / \mathbb{A}_{0}=0$. 
Energy relations for SH waves

585
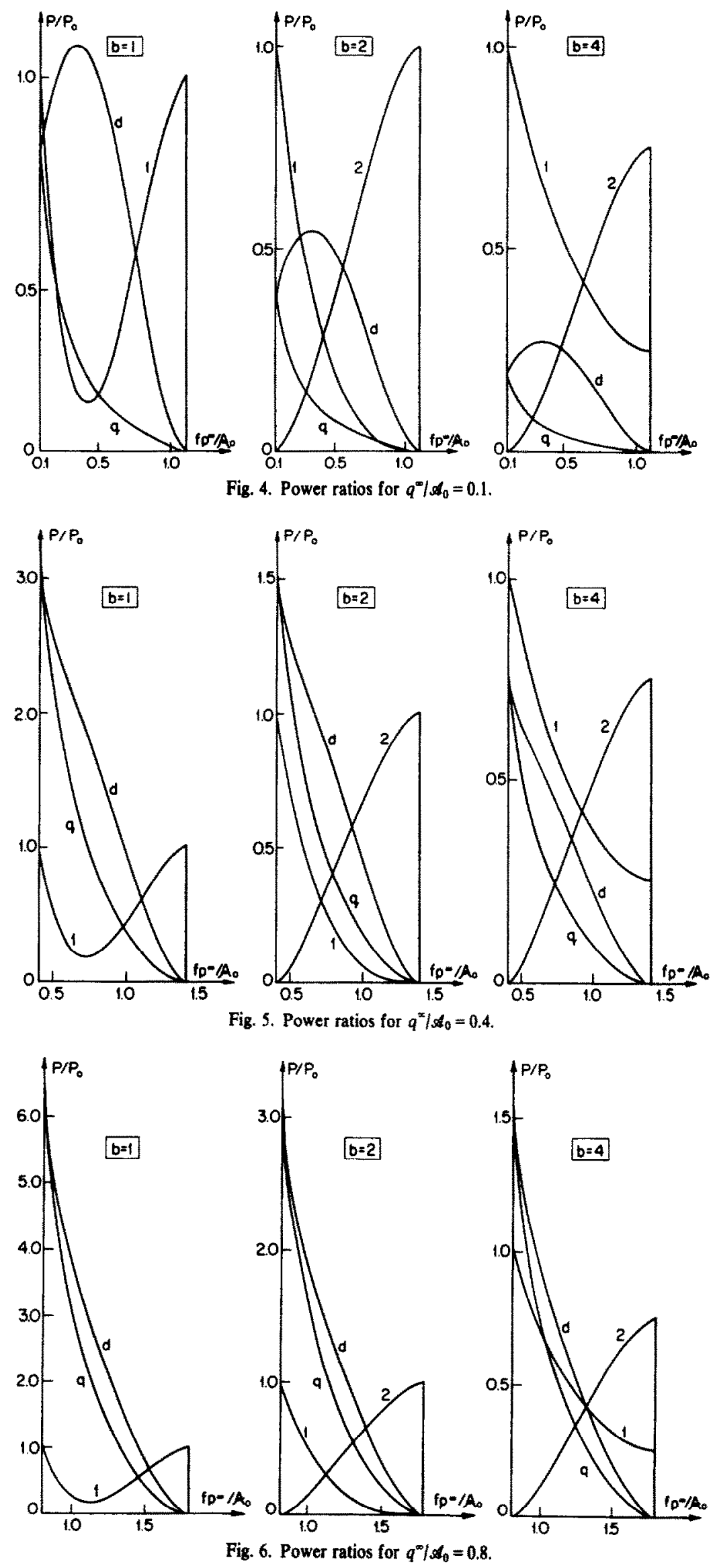
It is seen from the diagrams that the dissipated power peaks at intermediate values of $f p^{x} / \mathscr{A}_{0}$ when $q^{\infty} / \mathscr{A}_{0}$ is small, but that the dissipation peak shifts to the smallest possible value of $f p^{\infty} / \mathscr{A}_{0}$ when $q^{\infty} / \mathscr{A}_{0}$ is increased. A notable feature of the results is that in all cases $P_{d}>P_{q}$ which means the reflected and refracted waves never carry more energy than the incident wave, meaning that none of the work done by the applied tractions is transferred to the waves.

Acknowledgement-Support by the National Science Foundation under the grant CME 8019281 is gratefully acknowledged.

\section{REFERENCES}

1. E. L. Chez, J. Dundurs and M. Comninou, Bull. Seismol. Soc. Am. 68, 999 (1978).

2. M. Comninou, J. Dundurs and E. L. Chez, J. Acoust. Soc. Am. 66, 789 (1979).

3. M. Comninou and J. Dundurs, Int. J. Solids Structures 16, 283 (1980).

4. R. K. Miller, J. Appl. Mech. 4, 652 (1977).

5. R. K. Miller, Bull. Seismol. Soc. Am. 68, 987 (1978).

6. R. K. Miller and H. T. Tran, J. Appl. Mech 46, 625 (1979).

7. J. D. Achenbach, Wave Propagation in Elastic Solids. North Holland, Amsterdam (1973). 